\title{
Concept of Hokkaido University Picture Archiving Communication System
}

\author{
Goro Irie
}

\begin{abstract}
The basic concept of Hokkaido University picture archiving communication system (HU-PACS) for its design in hardware architecture and in its characteristic functions is reported. After almost 10 years, the concept was solidified as the opinion of most of the doctors of $\mathrm{HU}$ Hospital. Discussions with vendors were very helpful in providing technical or industrial expertise and equipment to effect this concept with the limited funds available. Copyright (C) 1991 by W.B. Saunders Company
\end{abstract}

KEY WORDS: HU-PACS, HU-MIS, HIS, film management, digital imaging.

$A$ FTER NEARLY 10 years of consideration, discussions, and experiments, the main part of Hokkaido University picture archiving communication system (HU-PACS) was implemented in January 1989. HU-PACS is a totally digital system resulting from technical progress in those 10 years in the fields of computed radiography using imaging plate (CR), optical disk, optical fiber, and related network technologies. Although the system does not yet cover the entire hospital (at present, it covers the radiology department and the orthopedic surgery and general medicine clinics), the system handles more than $50 \%$ of images produced daily in the hospital and its image quality on cathode-ray tube (CRT) and access efficiency are well accepted by the most of the doctors using the system for the months since its installation.

\section{MOTIVATION FOR AND GOAL OF HU-PACS}

The enormous pressure of the ever-growing amount of x-ray film that must be kept safely and managed efficiently for access by any doctor of the hospital made us consider PACS rather than a microfilm system or $\mathrm{x}$-ray film retrieval machines.

The amount of $x$-ray film used in the hospital is approximately 500,000 sheets each year, of which about $40 \%$ is generated by radiology and more than $15 \%$ is generated by orthopedic surgery. About $40 \%$ of images printed on film are distributed to the 18 other hospital departments, which keep the films independently. Although our film management is not centralized, the amount of film for the radiology and orthopedic surgery department became so great as to exceed their capabilities to manage the $\mathrm{x}$-ray film by themselves. The same type of catastrophe in film management is also anticipated sooner or later in many other departments if we lack a good image management system like PACS.

During the planning phase of PACS, we determined that PACS should handle not only $\mathrm{x}$ ray images but also all kinds of medical images, ie, scitigrams, echograms, colorphotograms of fiberscope, microscope, and retinograms, electroencephalograms, electrocardiograms, and even written medical records. By setting the goal of the PACS in this way, complicated and unclear discussions on cost performance can be avoided, because such a PACS is so valuable in medicine that to count its performance in terms of money would be meaningless. A PACS of this kind is furthermore very acceptable to everyone in the hospital because almost all doctors in the hospital can share the benefits of PACS.

Achieving consensus among doctors in the hospital is essential to implementation of an expensive system like PACS; without such consensus, the potentially good performance of such a system could not be realized even after the system was implemented. The goal of the HU-PACS, therefore, was set to include all medical imaging as described and to cover the entire hospital with its network and terminals. HU-PACS today is in its initial phase only; much more is expected of it in the future.

\section{System Configuration and Data Flow}

Conventional $\mathrm{x}$-ray film is to be almost completely replaced by an FCR system with 12 readout machines by March 1991; we are now in the second year of a three-year project. About twothird of our $\mathrm{x}$-ray images, therefore, are now taken

From the Department of Radiology and Division of Medical Information, Hokkaido University, Sapporo, Hokkaido, Japan.

Address reprint requests to Goro Irie, MD, Department of Radiology, Division of Medical Information, Hokkaido University, Kita 12 Jyo Nishi 3 Chrome, Kita-ku, Sapporo, Hokkaido, Japan.

Copyright (C) 1991 by W.B. Saunders Company

0897-1889/91/0404-0106\$03.00/0 
by an FCR system; of these images, only those ordered from the departments in which image terminals are installed are sent to the PACS. Five readout units of FCR, and 3 computed tomography $(C T)$ scans, and 1 magnetic resonance imaging (MRI) scan are connected on line to the PACS computers which are so-called supermini computers to manage image database in two jukebox type optical disk files of 48 Gbyte each and magnetic disk files of 4.8 Gbyte which serve as temporary but fast storage.

PACS computers are connected to an optical loop LAN of $100 \mathrm{Mbps}$ to send images to the image terminal from which the image was accessed. PACS computers are also connected by the optical loop LAN to two mainframe computers which manage a hospital information system (HIS) that has 19 Gbyte magnetic disk for its own data base, including the data of image IDs, and its own optical loop LAN and $350 \mathrm{HIS}$ terminals covering the entire hospital.

Thus far, we have described the characteristic hardware features of the HU-PACS (Fig 1) as a part of the HU Medical Information System (HUMIS). The innermost optical loop LAN(OLL) and optical discfile (ODF) attached to MIS host computers are not yet installed; these await the budget allocation for the medical record system. Four image workstations (IWS) with two CRTs and 16 image display terminals (IDT) with a single CRT are connected to the OLL by either net.

\section{Functions of the HU-PACS}

The characteristic functions of HU-PACS are coupling to HIS, data compression, and image processing. Coupling to HIS makes PACS computers free of data base management on image IDs and makes possible automated prefetching to the terminal or downloading to the faster media from the optical disk file beforehand for patients whose date of visit is known through the HIS booking system. Discrete Cosine Transform (DCT) method is adopted for data compression whose program is implemented on a hardware chip that achieves both processing time of data compression and its reverse about 1 second each.

Without the data compression of one tenth on the average, the ability of the HU-PACS would be much less than it should be; ie, the cost of the PACS would be unaffordable. Image processing

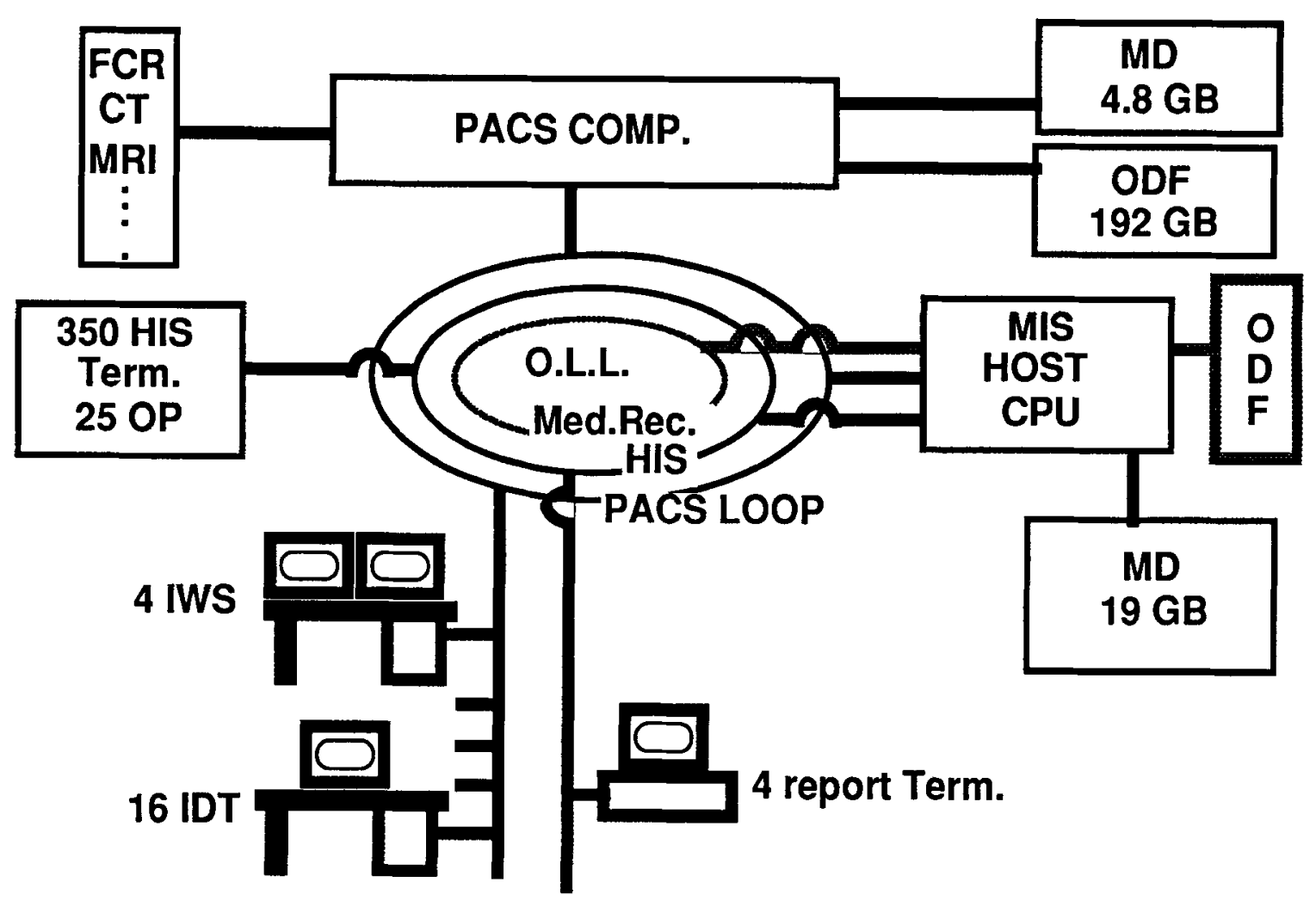

Fig 1. Configuration of HU-MIS. 
programs implemented on each terminal are very important because such programs allow the users to visualize the CRT image better than the film images, whose processing parameters, if any, are fixed and unchangeable for the users.

\section{Reporting System}

Most $\mathrm{x}$-ray images except those in teaching files should be abandoned after 10 to 20 years because images cannot be kept forever, no matter how great the improvements in recording media or compression technique. Medical findings should be extracted from the images repeatedly while they are kept in the PACS to form a data base of the findings along with their final diagnosis. This process is most easily performed by a good reporting system. Such a reporting system has been developed in our department. When opened, the system asks the user for the patient's identification (ID), the type of examination, and technical factors. Next, the system asks the user for each anatomic image finding conversationally. The user (radiologist) answers the questions using only number keys.

Using prepared sentences, the system prints out a report after the conversation. At the same time, the system updates the data base of the finding and calculates the estimated diagnosis by means of the most likely estimation. In this way, image information becomes a knowledge base. Teaching files of typical images must still be maintained properly. Candidate images for teaching files should be marked by users whenever the images are used for diagnosis in HUPACS.

\section{Application of PACS to Planning of Radiation Therapy}

We implemented HU-PACS with a strong belief that it should not be a doctors' toy but should show usefulness in patient care from the start. One evidence of this attitude is the linkage of the PACS to one of the three CTs that are a part of a three-dimensional radiotherapy planning system.

Through the PACS network or by floppy disc, the radiotherapist can refer to other images, ie,
FCR. MRI, single-photon emission CT (SPECT); angiography, gastrointestinal series, and/or echography, which may add useful information to the CT-aided treatment planning. An imageprocessing computer that is also a part of the planning system can magnify or rotate the image continuously to replace or overlap the referent image on the CT image of the planning system.

How much the results of radiation therapy are to be improved by this connection of HU-PACS and the planning system is not yet clear. It is, however, clear enough that this connection has given radiotherapists more confidence in their treatment planning, which more often results in multiportal irradiation with smaller fields than were used previously. Good results in tumor cure and less radiation damage to normal tissues are expected.

\section{CONCLUSIONS}

Owing to much recent technical progress, HUPACS has been implemented as a totally digital system. Data compression of about one-tenth on the average, however, is still necessary if HUPACS is to be reasonable in cost, size, and efficiency. Many other software packages help HUPACS to be a useful clinical tool. Characteristics of the hardware configuration of the PACS are (1) adoption of loop LAN of $100 \mathrm{Mbps}$ optical fiber with its branching bus-type LANs, (2) coupling with HIS which enables downloading and prefetching of images for a booked patient, (3) taking all radiographic images by an FCR system, which no film and film digitizer and (4) on-line connection to a three-dimensional radiotherapy planning system.

Our final goal for the HU-PACS is far in the future because we are aiming at a large-scale PACS that handles not only radiological images but also all medical images in their widest scope as well as the entire hospital with no exception. To set the goal of the PACS at this level and to achieve successful implementation of HU-PACS, we believed it very important to have unanimous agreement and good support from all hospital personnel. 